\title{
Effect of Rotation, Magnetic Field and Initial Stresses on Propagation of Plane Waves in Transversely Isotropic Dissipative Half Space
}

\author{
Sushant Shekhar ${ }^{1,2}$, Imtiyaz A. Parvez ${ }^{1,2}$ \\ ${ }^{1}$ Academy of Scientific and Innovative Research, New Delhi, India \\ ${ }^{2}$ CSIR Center for Mathematical Modelling and Computer Simulation, NAL Belur Campus, Bangalore, India \\ Email: sushant.shekhar85@gmail.com,parvez@cmmacs.ernet.in
}

Received May 13, 2013; revised June 13, 2013; accepted June 20, 2013

Copyright (C) 2013 Sushant Shekhar, Imtiyaz A. Parvez. This is an open access article distributed under the Creative Commons Attribution License, which permits unrestricted use, distribution, and reproduction in any medium, provided the original work is properly cited.

\begin{abstract}
The problem regarding the reflection of plane waves in a transversely isotropic dissipative medium is considered, in which we are studying about the reflection of incidence waves in initially stressed dissipative half space. After solving the governing equations, we find the two complex quasi- $P(q P)$ and quasi- $S V(q S V)$ waves. The occurrence of reflected waves is studied to calculate the reflection coefficient and the energy partition of incidence wave at the plane boundary of the dissipative medium. Numerical example is considered for the reflection coefficient and the partition of incident energy, in which we study about the effect of rotation, initial stresses and magnetic field.
\end{abstract}

Keywords: Magnetic Field; Initial Stress; Rotation; Reflection of Plane Waves; Reflection Coefficients; Energy Ratios

\section{Introduction}

In recent years, most of the researchers are interested to solve the problems related to electrically conducting elastic media permeated by uniform magnetic fields. The seismic wave propagation has been used for various studies related to magneto-elasticity on the Earth's mantle and cores. By the knowledge related to reflection and refraction, the plane waves are the source of information used to image the Earth's interior. Dissipation of the medium depends upon the internal structure. The initial stress in the medium may be developed due to some reasons such as slow process of creep, gravity, external forces, difference in temperature, etc.

Problem related to plane waves in transversely isotropic medium is very important for the possible application in various branches of science and technology such as earthquake science, acoustic, geophysics and optics etc. Biot [1] observed that the initial stresses have notable effect on the propagation on elastic waves in a medium. The problem related to reflection and refraction of elastic waves from the boundaries of different media has been discussed in the famous book by Achenbach [2]. Borejko [3] introduced the reflection and transmission coefficients for three dimensional plane waves in elastic media. By using the Biot's theory [4] several researchers [5-9] have studied extensively the propagation of elastic waves. The problem related to reflection and refraction of $q P$ and $q S V$ waves at the interface of Fiber-reinforced medium has been discussed by Chattopadhyay and Venkateswarlu [10]. A huge amount of mathematical work has been performed for the propagation of elastic waves in dissipative medium; see for instance [11-14]. The problem related to thermo-magneto-dynamic stresses and perturbation of magnetic field vector in a non-homogeneous hollow cylinder has been studied by Kong et al. [15].

In the present study, we have used the Biot's incremental deformation theory [4] for deriving the algebraic expressions of the reflection coefficients and energy ratios when plane waves of $q P$ and $q S V$ type are incident on the initially stressed dissipative half-space with stress free boundaries. The dispersion equations for reflection coefficients and energy ratios of incident $q P$ and $q S V$ waves on the free surface of the initially stressed dissipative medium have been derived and observed that in presence of initial stresses, magnetic field and rotation of the medium must affect the reflection coefficients and energy shares of reflected plane waves. 


\section{Formulation of the Problem}

We consider the medium is a perfect electric conductor. The Maxwell's equations of electromagnetic field for perfectly conducting elastic medium are given by Raychoudhuri and Banerjee [7].

$$
\begin{aligned}
& \nabla \times \boldsymbol{h}=\boldsymbol{J}, \nabla \times \boldsymbol{E}=-\mu_{e} \frac{\partial \boldsymbol{h}}{\partial t}, \\
& \nabla \cdot \boldsymbol{h}=0, \nabla \cdot \boldsymbol{E}=0, \\
& \boldsymbol{h}=\nabla \times(\boldsymbol{u} \times \boldsymbol{H}), \\
& \boldsymbol{H}=\boldsymbol{H}_{0}+\boldsymbol{h}
\end{aligned}
$$

where, $\nabla$ is the Hamilton's operator, $\mu_{0}$ is the magnetic permeability, $\boldsymbol{J}$ is the current density vector, $\boldsymbol{E}$ and $\boldsymbol{h}=(0, h, 0)$ are the induced electric field and the induced magnetic field both are developed due to application of initial magnetic field $\boldsymbol{H}_{0}=\left(0, H_{0}, 0\right)$.

We consider a transversely isotropic dissipative half space rotating about y-axis $\boldsymbol{\Omega}=(0, \Omega, 0)$ under constant magnetic field (along y-axis) and initial compressive stress $P$ along $\mathrm{x}$-axis (Figure 1).

The equations of motion in $x-z$ plane for the present problem may be written in the forms, given by Biot $[1,4]$

$$
\begin{aligned}
& \frac{\partial s_{11}}{\partial x}+\frac{\partial s_{13}}{\partial z}-P \frac{\partial \omega}{\partial z}+\mu_{e} H_{0}^{2}\left(\frac{\partial^{2} u}{\partial x^{2}}+\frac{\partial^{2} w}{\partial x \partial z}\right) \\
= & \rho\left(\frac{\partial^{2} u}{\partial t^{2}}-\Omega^{2} u+2 \Omega \frac{\partial w}{\partial t}\right), \\
& \frac{\partial s_{31}}{\partial x}+\frac{\partial s_{33}}{\partial z}-P \frac{\partial \omega}{\partial x}+\mu_{e} H_{0}^{2}\left(\frac{\partial^{2} u}{\partial x \partial z}+\frac{\partial^{2} w}{\partial z^{2}}\right) \\
= & \rho\left(\frac{\partial^{2} w}{\partial t^{2}}-\Omega^{2} w-2 \Omega \frac{\partial u}{\partial t}\right)
\end{aligned}
$$

where $\rho$ is density, $\omega=\frac{1}{2}\left(\frac{\partial w}{\partial x}-\frac{\partial u}{\partial z}\right)$ is the rotational component and $s_{i j}(i, j=1,3)$ are incremental stress tensor in $x-z$ plane.

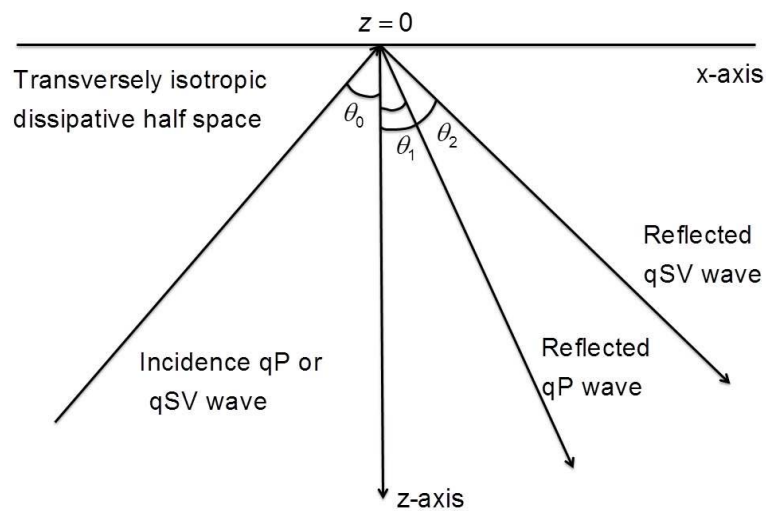

Figure 1. Geometry of the problem.
The stress-strain relation for transversely isotropic medium can be given by Biot [1]

$$
\begin{aligned}
& s_{11}=\left(c_{11}+P\right) e_{11}+\left(c_{13}+P\right) e_{33} \\
& s_{13}=s_{31}=c_{44} e_{13} \quad s_{33}=c_{13} e_{11}+c_{33} e_{33}
\end{aligned}
$$

where $c_{i j}$ are the elastic coefficients.

For dissipative medium, the elastic coefficients must be of complex constants, can be written as

$$
\begin{aligned}
& c_{11}=c_{11}^{R}+\mathrm{i} c_{11}^{I}, \quad c_{13}=c_{13}^{R}+\mathrm{i} c_{13}^{I} \\
& c_{33}=c_{33}^{R}+\mathrm{i} c_{33}^{I}, \quad c_{44}=c_{44}^{R}+\mathrm{i} c_{44}^{I}
\end{aligned}
$$

where $\mathrm{i}=\sqrt{-1}, \quad c_{11}^{R}, c_{13}^{R}, c_{33}^{R}, c_{44}^{R}, c_{11}^{I}, c_{13}^{I}, c_{33}^{I}, c_{44}^{I}$, are all real constants.

The incremental strain components $e_{i j}(i, j=1,3)$ are related to the displacement components $(u, w)$ which are given by the relation

$$
e_{11}=\frac{\partial u}{\partial x}, e_{33}=\frac{\partial w}{\partial z}, e_{13}=\left(\frac{\partial w}{\partial x}+\frac{\partial u}{\partial z}\right)
$$

Let the harmonic solution of Equation (2), for the propagation of plane waves, the displacement components are written as follows:

$$
\left(u^{(n)}, w^{(n)}\right)=\left(A_{n} d_{1}^{(n)}, A_{n} d_{3}^{(n)}\right) \mathrm{e}^{\mathrm{i} k_{n}\left(x p_{1}^{(n)}+z p_{3}^{(n)}-v_{n} t\right)}
$$

Where the index $n$ is assigns an arbitrary direction of propagation of the plane waves. $p_{1}^{(n)}$ and $p_{3}^{(n)}$ are cosine of angle made by direction of propagation with the normal (z-axis), $\left(d_{1}^{(n)}, d_{3}^{(n)}\right)$ are the component of unit displacement vector, $A_{n}$ is the arbitrary constant and $v_{n}$ and $k_{n}$ are the velocity of propagation and corresponding wave number.

Substituting Equation (6) in (2) with the help of Equations (3)-(5) then we get the velocities correspond to quasi- $P(q P)$ and quasi-SV $(q S V)$ wave respectively.

$$
\begin{aligned}
& {\left[L_{1}-\rho v_{n}^{2}\left\{1+\left(\frac{\Omega}{\omega}\right)\right\}\right] A_{n} d_{1}^{(n)}+\left[L_{2}-2 \mathrm{i} \rho v_{n}^{2}\left(\frac{\Omega}{\omega}\right)\right] A_{n} d_{3}^{(n)} } \\
= & 0 \\
& {\left[L_{2}+2 \mathrm{i} \rho v_{n}^{2}\left(\frac{\Omega}{\omega}\right)\right] A_{n} d_{1}^{(n)}+\left[L_{3}-\rho v_{n}^{2}\left\{1+\left(\frac{\Omega}{\omega}\right)\right\}\right] A_{n} d_{3}^{(n)} } \\
= & 0
\end{aligned}
$$

For non-trivial solution of Equations (7) and (8), the determinant must be equal to zero. That gives two values of $v_{n}^{2}$, which are given by

$$
v_{n}^{2}=v_{p}^{2}=\frac{\left(L_{1}+L_{3}\right)+\sqrt{\left(L_{1}-L_{3}\right)^{2}+4 L_{2}^{2}}}{2 \rho\left\{1+\left(\frac{\Omega}{\omega}\right)^{2}\right\}}
$$




$$
v_{n}^{2}=v_{S V}^{2}=\frac{\left(L_{1}+L_{3}\right)-\sqrt{\left(L_{1}-L_{3}\right)^{2}+4 L_{2}^{2}}}{2 \rho\left\{1+\left(\frac{\Omega}{\omega}\right)^{2}\right\}}
$$

where

$$
\begin{aligned}
L_{1}= & \left(c_{11}^{R}+P+\mu_{e} H_{0}^{2}\right) p_{1}^{(n)^{2}}+\left(c_{44}^{R}+\frac{P}{2}\right) p_{3}^{(n)^{2}} \\
& +\mathrm{i}\left(c_{11}^{I} p_{1}^{(n)^{2}}+c_{44}^{I} p_{3}^{(n)^{2}}\right), \\
L_{2}= & \left(c_{13}^{R}+c_{44}^{R}+\mu_{e} H_{0}^{2}+\frac{P}{2}\right) p_{1}^{(n)} p_{3}^{(n)}+\mathrm{i}\left(c_{13}^{I}+c_{44}^{I}\right) p_{1}^{(n)} p_{3}^{(n)}, \\
L_{3}= & \left(c_{44}^{R}-\frac{P}{2}\right) p_{1}^{(n)^{2}}+\left(c_{33}^{R}+\mu_{e} H_{0}^{2}\right) p_{3}^{(n)^{2}} \\
& +\mathrm{i}\left(c_{44}^{I} p_{1}^{(n)^{2}}+c_{33}^{I} p_{3}^{(n)^{2}}\right)
\end{aligned}
$$

The real and imaginary parts of Equations (9) and (10) represents the phase velocities and damping velocities of $q P$ and $q S V$ waves, respectively. From the Equations (9) and (10), we can say that both $v_{P}$ and $v_{S V}$ depend on initial stresses, rotation, magnetic field, damping and direction of propagation $\left(p_{1}^{(n)}, p_{2}^{(n)}\right)$. Also relation between the unit displacement vectors can be given as

$$
\frac{d_{1}^{(n)}}{d_{3}^{(n)}}=-\frac{L_{2}-2 \mathrm{i} \rho v^{2}\left(\frac{\Omega}{\omega}\right)}{L_{1}-\rho v^{2}\left\{1+\left(\frac{\Omega}{\omega}\right)^{2}\right\}}=-\frac{L_{3}-\rho v^{2}\left\{1+\left(\frac{\Omega}{\omega}\right)^{2}\right\}}{L_{2}+2 \mathrm{i} \rho v^{2}\left(\frac{\Omega}{\omega}\right)} .
$$

\section{Reflection of Plane Waves from Stress Free Surface}

We consider an initially stressed rotating magneto-elastic transversely isotropic dissipative half space absorbing the region $z \geq 0$. In this section, we are discussing about the reflection coefficients for incident $q P$ and $q S V$ waves.

The displacement components of solid particles in transversely isotropic dissipative medium due to presence of an incidence wave and reflected waves are expressed as follows:

$$
\begin{aligned}
u(x, z, t)= & A_{0} d_{1}^{(0)} \exp \left\{\mathrm{i} k\left(p_{1}^{(0)} x_{1}+p_{3}^{(0)} x_{3}-v_{0} t\right)\right\} \\
& +\sum_{j=1}^{2} A_{j} d_{1}^{(j)} \exp \left\{\mathrm{i} k\left(p_{1}^{(j)} x_{1}+p_{3}^{(j)} x_{3}-v_{j} t\right)\right\}, \\
w(x, z, t)= & A_{0} d_{3}^{(0)} \exp \left\{\mathrm{i} k\left(p_{1}^{(0)} x_{1}+p_{3}^{(0)} x_{3}-v_{0} t\right)\right\} \\
& +\sum_{j=1}^{2} A_{j} d_{3}^{(j)} \exp \left\{\mathrm{i} k\left(p_{1}^{(j)} x_{1}+p_{3}^{(j)} x_{3}-v_{j} t\right)\right\}
\end{aligned}
$$

We are assuming the superscript $(0)$ for incident $q P$ and $q S V$ waves, (1) for reflected $q P$ waves and (2) for reflected $q S V$ waves.

The boundary conditions for the free surfaces are vanishing of incremental boundary forces. So, the two boundary conditions required to be satisfied at the plane $z=0$, are

$$
\Delta f_{x}=s_{13}^{(n)}+e_{13}^{(n)} P=0, \Delta f_{z}=s_{33}^{(n)}=0
$$

Equation (13) can be written as

$$
\begin{aligned}
& s_{13}^{(0)}+s_{13}^{(1)}+s_{13}^{(2)}+\left(e_{13}^{(0)}+e_{13}^{(1)}+e_{13}^{(2)}\right) P=0, \\
& s_{33}^{(0)}+s_{33}^{(1)}+s_{33}^{(2)}=0
\end{aligned}
$$

Equation (12) will satisfy the boundary conditions (14), if the following Snell's law holds

$$
\frac{p_{1}^{(0)}}{v_{0}}=\frac{p_{1}^{(1)}}{v_{1}}=\frac{p_{1}^{(2)}}{v_{2}}
$$

with the relations

$$
\begin{aligned}
& A_{0} \delta_{0}+A_{1} \delta_{1}+A_{2} \delta_{2}=0 \\
& A_{0} \delta_{3}+A_{1} \delta_{4}+A_{2} \delta_{5}=0
\end{aligned}
$$

where

$$
\begin{aligned}
& \delta_{0}=k_{0} L\left(p_{3}^{(0)} d_{1}^{(0)}+p_{1}^{(0)} d_{3}^{(0)}\right), \\
& \delta_{1}=k_{1} L\left(p_{3}^{(1)} d_{1}^{(1)}+p_{1}^{(1)} d_{3}^{(1)}\right), \\
& \delta_{2}=k_{2} L\left(p_{3}^{(2)} d_{1}^{(2)}+p_{1}^{(2)} d_{3}^{(2)}\right), \\
& \delta_{3}=k_{0}\left(c_{33} p_{3}^{(0)} d_{3}^{(0)}+c_{13} p_{1}^{(0)} d_{1}^{(0)}\right), \\
& \delta_{4}=k_{1}\left(c_{33} p_{3}^{(1)} d_{3}^{(1)}+c_{13} p_{1}^{(1)} d_{1}^{(1)}\right), \\
& \delta_{5}=k_{2}\left(c_{33} p_{3}^{(2)} d_{3}^{(2)}+c_{13} p_{1}^{(2)} d_{1}^{(2)}\right), \\
& L=c_{44}+\frac{P}{2}
\end{aligned}
$$

The amplitude ratios for incidence $q P$ and $q S V$ waves can be given by

$$
\frac{A_{1}}{A_{0}}=-\frac{\delta_{0} \delta_{5}-\delta_{3} \delta_{2}}{\delta_{1} \delta_{5}-\delta_{4} \delta_{2}}, \quad \frac{A_{2}}{A_{0}}=-\frac{\delta_{0} \delta_{4}-\delta_{3} \delta_{1}}{\delta_{2} \delta_{4}-\delta_{5} \delta_{1}}
$$

The real part of Equation (18) allow to determine the reflection coefficients of the reflected $q P$ and $q S V$ waves at a given incident $q P$ and $q S V$ waves. The reflection coefficients are denoted by $Z_{P P}$ (the incident $q P$ wave is reflected as the $q P$ wave), $Z_{P S}$ (the incident $q P$ wave is reflected as the $q S V$ wave), $Z_{S P}$ (the incident $q S V$ wave is reflected as the $q P$ wave) and $Z_{S S}$ (the incident $q S V$ wave is reflected as the $q S V$ wave).

\section{Energy Ratios}

We are considering the surface element of unit area at the 
half space $z \geq 0$. Our main purpose is to calculate the distribution of energy (of the incident wave) among the two reflected waves on this half space. Following Achenbach [2], the rate at which the incident energy transmitted per unit area is given by the scalar product of surface traction and particle velocity denoted by $P^{*}$. The time average of $P^{*}$ over a period, denoted by $\left\langle P^{*}\right\rangle$, represents the average energy transmission per unit area per unit time.

The average energy flux, for a given surface with normal along $z$-direction is represented through the components $\left\langle P^{*}\right\rangle$ is given by

$$
\left\langle P_{i j}^{*}\right\rangle=\mathfrak{R}\left(s_{31}^{(i)}\right) \mathfrak{R}\left(\dot{u}_{1}^{(j)}\right)+\mathfrak{R}\left(s_{33}^{(i)}\right) \mathfrak{R}\left(\dot{u}_{3}^{(j)}\right)
$$

The distribution of incident energy is given in the form of a matrix at the free surface of the dissipative medium.

$$
E_{i j}=-\frac{\mathfrak{R}\left\langle P_{i j}^{*}\right\rangle}{\mathfrak{R}\left\langle P_{00}^{*}\right\rangle}, \quad(i, j=0,1,2)
$$

The element of matrix $\boldsymbol{P}$ is defined as follows:

$$
\begin{aligned}
\left\langle P_{i j}\right\rangle=A_{i} \bar{A}_{j} & \left\{\left(d_{i}^{(1)} p_{3}^{(i)}+d_{i}^{(3)} p_{1}^{(i)}\right) c_{44} \bar{d}_{j}^{(1)}\right. \\
& \left.+\left(c_{13} d_{i}^{(1)} p_{1}^{(i)}+c_{33} d_{i}^{(3)} p_{3}^{(i)}\right) \bar{d}_{j}^{(3)}\right\} \bar{v}_{j} k_{i} \bar{k}_{j}
\end{aligned}
$$

where bar over entity implies complex conjugate. The sum of all non-diagonal entries of this matrix calculates the share of interaction energy in the medium. The diagonal entries $E_{11}$ and $E_{22}$ of the matrix (Equation (20)) denote the energy shares of reflected $q P$ and $q S V$ waves in the incident energy. The energy due to interaction between incident wave and two reflected waves is given by $E_{I R}=\sum_{i=1}^{2}\left(E_{0 i}+E_{i 0}\right)$. The energy due to the interaction among the two reflected waves is given by $E_{R R}=\sum_{i=1}^{2}\left(\sum_{j=1}^{2} E_{i j}-E_{i i}\right)$. The conservation of the energy at the free-surface is given by the relation.

$$
E_{11}+E_{22}+E_{I R}+E_{R R}=1
$$

\section{Numerical Results and Discussion}

Material parameter for copper-alloy is chosen for the numerical model of magneto-elastic medium. The elastic and dynamic constants are given by $[8,11]$

$$
\begin{array}{ll}
c_{11}^{R}=2.628 \times 10^{10} \mathrm{~N} \cdot \mathrm{m}^{-2}, & c_{33}^{R}=1.562 \times 10^{10} \mathrm{~N} \cdot \mathrm{m}^{-2}, \\
c_{13}^{R}=0.508 \times 10^{10} \mathrm{~N} \cdot \mathrm{m}^{-2}, & c_{33}^{R}=0.385 \times 10^{10} \mathrm{~N} \cdot \mathrm{m}^{-2}, \\
c_{11}^{I}=1.025 \times 10^{10} \mathrm{~N} \cdot \mathrm{m}^{-2}, & c_{33}^{I}=0.950 \times 10^{10} \mathrm{~N} \cdot \mathrm{m}^{-2}, \\
c_{13}^{I}=0.425 \times 10^{10} \mathrm{~N} \cdot \mathrm{m}^{-2}, & c_{33}^{I}=0.325 \times 10^{10} \mathrm{~N} \cdot \mathrm{m}^{-2}, \\
\rho=7.14 \times 10^{3} \mathrm{~kg} \cdot \mathrm{m}^{-3}, & \mu_{e} H_{0}^{2}=1.24 \times 10^{9} \mathrm{~N} \cdot \mathrm{m}^{-2} .
\end{array}
$$

The given values of various parameters are used to calculate the complex velocities ( $q P$ and $q S V$ waves) reflection coefficients $\left(Z_{P P}, Z_{P S}, Z_{S P}\right.$ and $\left.Z_{S S}\right)$ and energy shares $\left(E_{11}, E_{22}, E_{I R}\right.$ and $\left.E_{R R}\right)$ in the dissipative medium. The variation of reflection coefficients and Energy ratios with angle of incidence $(\theta)$ are shown in Figures 2-5 (for incidence $q P$ wave) and in Figures 6-9 (for incidence $q S V$ wave).
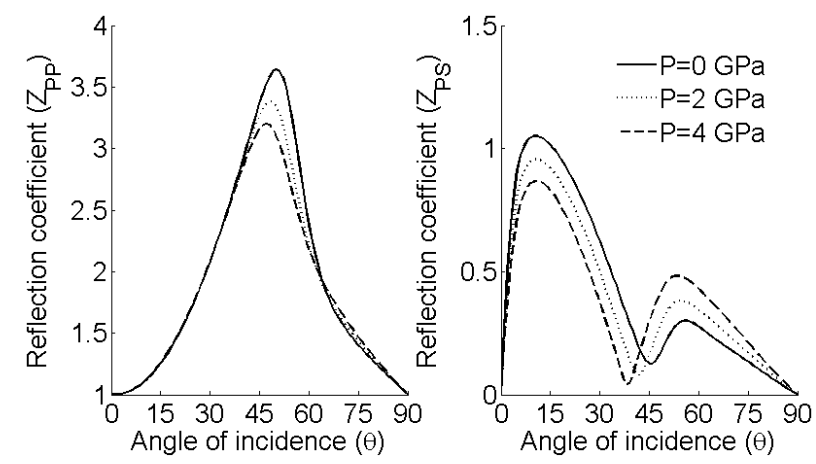

Figure 2. Reflection coefficients of $q P$ and $q S V$ waves with variations in incident angle $(\theta)$ and initial stress $P$ for incident $q P$ wave.
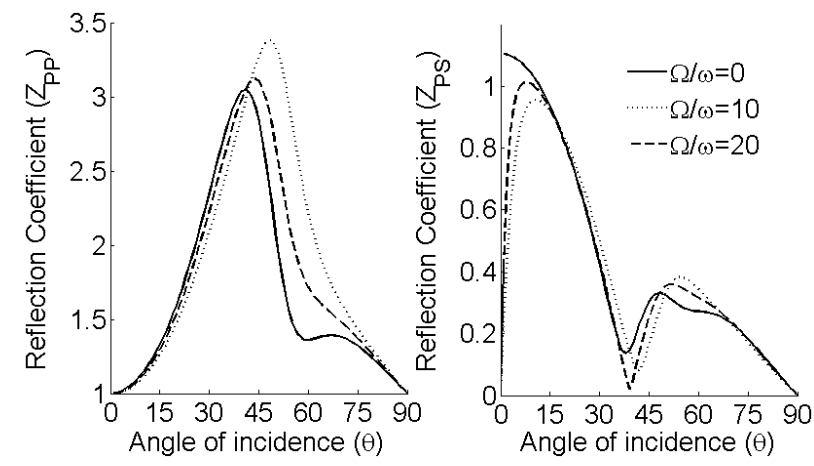

Figure 3. Reflection coefficients of $q P$ and $q S V$ waves with variations in incident angle $(\theta)$ and Effect of rotation parameter $(\Omega / \omega)$ for incident $q P$ wave.
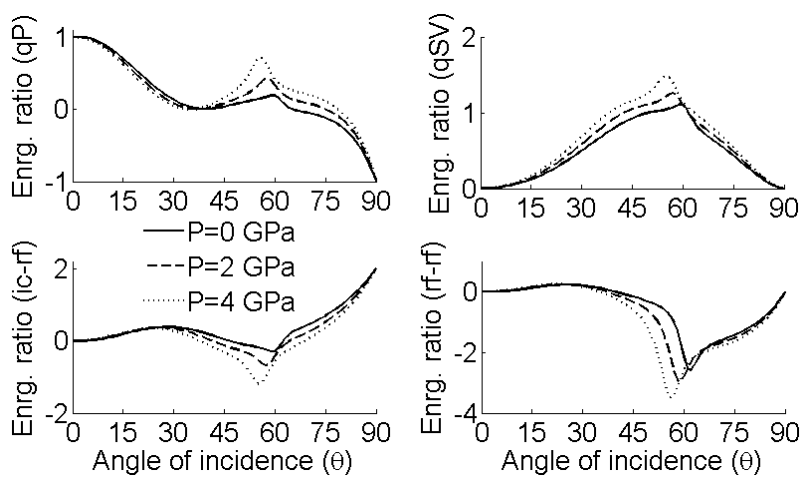

Figure 4. Energy shares of reflected $(q P, q S V)$ waves, incident and reflected waves interaction and interaction among reflected waves, variations with incident angle $(\theta)$ and Initial stress $(P)$ for incident $q P$ wave. 

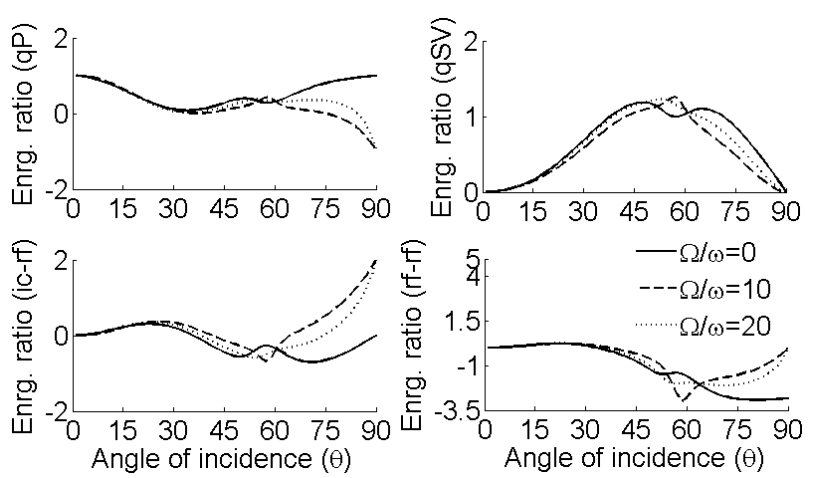

Figure 5. Energy shares of reflected $(q P, q S V)$ waves, incident and reflected waves interaction and interaction among reflected waves, variations with incident angle $(\theta)$ and rotational parameter $(\Omega / \omega)$ for incident $q P$ wave.
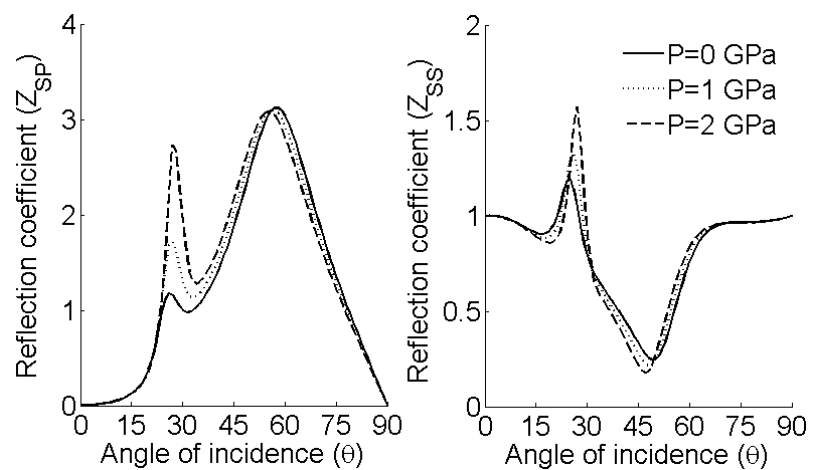

Figure 6. Reflection coefficients of $q P$ and $q S V$ waves with variations in incident angle $(\theta)$ and initial stress $(P)$ for incident $q S V$ wave.
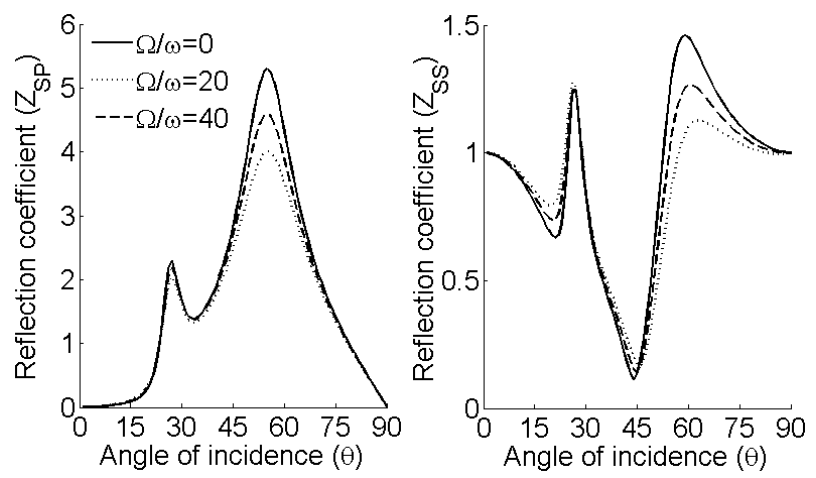

Figure 7. Reflection coefficients of $q P$ and $q S V$ waves with variations in incident angle $(\theta)$ and Effect of rotation parameter $(\Omega / \omega)$ for incident $q S V$ wave.

Figure 2 shows that the variation in the reflection coefficients $\left(Z_{P P}\right.$ and $\left.Z_{P S}\right)$ with respect to angle of incidence $(\theta)$ for different values of initial stress $(P)$. The comparison of the solid and dashes lines shows that the reflection coefficients $Z_{P P}$ and $Z_{P S}$ change in presence of initial stresses for each angle of incidence of $q P$ wave except grazing incidence. The effect of initial stresses is mini-
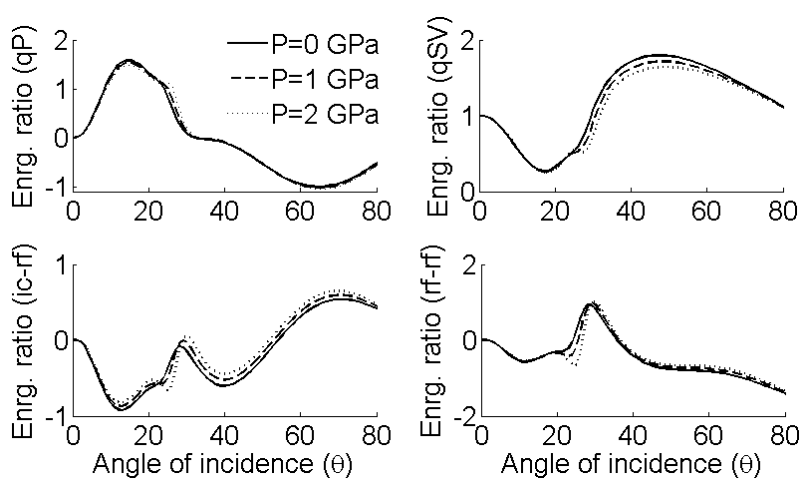

Figure 8. Energy shares of reflected $(q P, q S V)$ waves, incident and reflected waves interaction and interaction among reflected waves, variations with incident angle $(\theta)$ and Initial stress $(P)$ for incident $q S V$ wave.
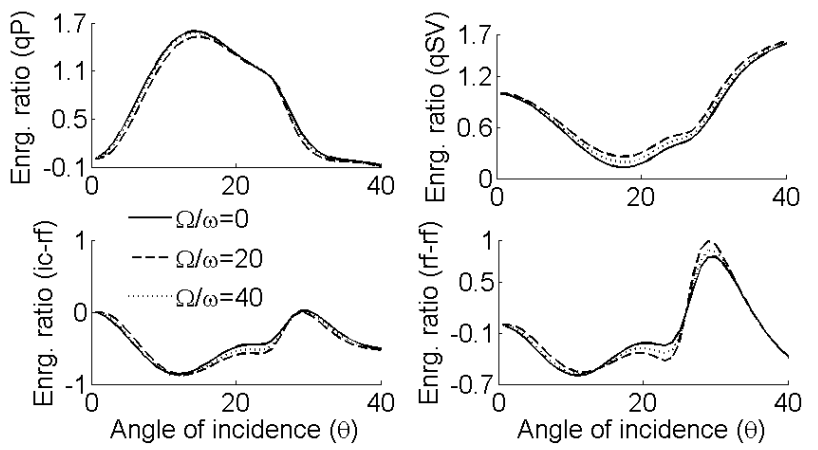

Figure 9. Energy shares of reflected $(q P, q S V)$ waves, incident and reflected waves interaction and interaction among reflected waves, variations with incident angle $(\theta)$ and rotational parameter $(\Omega / \omega)$ for incident $q S V$ wave.

mum in the range of $50^{\circ}<\theta<85^{\circ}$ for $Z_{P P}$ and $20^{\circ}<\theta<$ $80^{\circ}$ for $Z_{P S}$.

From Figure 3, it is noticed that $Z_{P P}$ and $Z_{P S}$ changes in the presence of rotation of the medium at each angle of the incidence of $q P$ wave except some grazing points.

The Figure 4 for incidence $q P$ wave shows that the energy variations with respect to angle of incidence $(\theta)$ for different values of initial stress $P$. The reflected $q P$ and $q S V$ waves reflect strongly in the presence of initial stress. We can see that as increase of initial stress, the reflected $q P$ wave reflects slowly in the range of $0^{\circ}<\theta<$ $30^{\circ}$ but the reflected $q S V$ wave reflects strongly in this range. For normal incidence, interaction between incidence wave and reflected waves is maximum whereas interaction between reflected waves is absent.

For the incidence of $q P$ wave, effect of different values of rotation parameter $(\Omega / \omega)$ on energy partition is shown in Figure 5. It is clear that at incident angle $\theta=0^{\circ}$ no effect of change in rotation parameter is shown. Also, both $q P$ and $q S V$ waves reflect weakly with increase the rotation parameter in the range $\left(60^{\circ}<\theta<90^{\circ}\right)$ The critical angle for incidence $q P$ wave is around $60^{\circ}$. 
From Figures 6 and 7, it can be easily seen that for the incidence of $q S V$ wave, the reflection coefficients $Z_{S P}=0$ and $Z_{S S}=1$ at the incidence angle $\theta=0^{\circ}$ and $\theta=90^{\circ}$ It shows that there is only one reflected $q P$ wave.

For the incidence of $q S V$ wave, the change in energy shares of reflected waves with change in initial stress is shown in Figure 8. The reflected $q P$ and $q S V$ waves are not more affected by the change in initial stress; the small change is shown in the range $\left(25^{\circ}<\theta<70^{\circ}\right)$ for $q S V$ wave. Also the change in initial stress affects the interaction between incidence and reflected waves in the range $\left(15^{\circ}<\theta<80^{\circ}\right)$ but very less for the interaction between reflected waves.

The Figure 9 for incidence $q S V$ wave shows that energy variations with respect to incident angle $(\theta)$ for different values on rotation parameter $(\Omega / \omega)$. As increasing the rotation parameter results is stronger the reflected $q S V$ wave but weaken the reflected $q P$ wave. For normal incidence, $q S V$ wave reflects strongly but $q P$ wave is almost absent.

A significant effect of the magnetic field on the propagation of plane waves in dissipative medium is observed by the comparison of our results with results of the study, see [13]. From, the comparison of Figure 2 with Figure $\mathbf{2}$ of the study, see [13], we find that no significant effect has been shown in the study, see [13] but in our results it is clear that how does initial stress affect the reflection coefficients. If, we neglect the effect of magnetic field then our results coincide with the study, see [13], except the energy ratios. From, the comparison of the Figure 3 with Figure 3 of the study, see [13], it is clear that in presence of magnetic field, the reflection coefficient decreases. Comparison of Figure 6 is made with Figure 4 of the study, see [13], the maximum value of reflection coefficients for angle of incidence in between $\left(0^{\circ}<\theta<\right.$ $45^{\circ}$ ) is around $\theta=30^{\circ}$ but it is obtained maximum at an angle $\theta=0^{\circ}$ for the study, see [13]. From the comparison of these results with the study, see [13], we find that physics of the problem will change in the presence of the magnetic field.

\section{Conclusion}

The reflection from the stress free surface of an initially stressed rotating magneto-elastic transversely isotropic dissipative medium is considered. The derived expression of reflection coefficients and energy ratios are obtained for incidence of $q P$ and $q S V$ waves. For copper alloy material, reflection coefficients and energy ratios are computed and presented graphically with angle of incidence for different values of initial stress and rotation parameter in presence of magnetic field. From the graphical representation it is observed that the presence of initial stresses, rotation parameter and magnetic field affect significantly to the reflection coefficients and energy ratios.

\section{Acknowledgements}

The authors convey their sincere thanks to Council of Scientific and industrial research (CSIR), New Delhi, for providing SRF to Mr. Sushant Shekhar and also thanks to Scientist in-charge CMMACS, Bangalore for providing all facilities related to this research work.

\section{REFERENCES}

[1] M. A. Biot, "The Influence of Initial Stress on Elastic Waves,” Journal of Applied Physics, Vol. 11, No. 8, 1940, pp. 522-530. doi:10.1063/1.1712807

[2] J. D. Achenbach, "Wave Propagation in Elastic Solids," North-Holland Pub. Co., New York, 1973.

[3] P. Borejko, "Reflection and Transmission Coefficients for Three-Dimensional Plane Waves in Elastic Media," Wave Motion, Vol. 24, No. 4, 1996, pp. 371-393. doi:10.1016/S0165-2125(96)00026-1

[4] M. A. Biot, "Mechanics of Incremental Deformation," Wiley, New York, 1965.

[5] S. Dey and D. Dutta, "Propagation and Attenuation of Seismic Body Waves in Initially Stressed Dissipative Medium,” Acta Geophysica, Vol. 46, No. 3, 1998, pp. 351366.

[6] R. W. Ogden and D. Sotirropoulos, "Reflection of Plane Waves from the Boundary of a Pre-Stressed Compressible Elastic Half-Space," IMA Journal of Applied Mathematics, Vol. 61, No. 1, 1998, pp. 61-90. doi:10.1093/imamat/61.1.61

[7] S. K. Roychoudhuri and S. Banerjee, "Magneto-Thermoelastic Interaction in an Infinite Viscoelastic Cylinder of Temperature-Rate Dependent Material Subjected to a Periodic Loading," International Journal of Engineering Science, Vol. 36, No. 5-6, 1998, pp. 635-643. doi:10.1016/S0020-7225(97)00096-7

[8] M. D. Sharma, "Effect of Initial Stress on Reflection at the Free Surfaces of Anisotropic Elastic Medium," Journal of Earth System Science, Vol. 116, No. 6, 2007, pp. 537-551. doi:10.1007/s12040-007-0049-8

[9] R. D. Tooly, T. W. Spencer and H. F. Sagoci, "Reflection and Transmission of Plane Compressional Waves," Geophysics, Vol. 30, No. 4, 1965, pp. 552-570. doi:10.1190/1.1439622

[10] A. Chattopadhyay and R. L. K. Venkateswarlu, "Reflection and Refraction of Quasi P and SV Waves at the Interface of Fiber-Reinforced Media,” Advanced Studies in Theoretical Physics, Vol. 1, No. 2, 2007, pp. 57-73.

[11] A. N. Norris, "Propagation of Plane Waves in a PreStressed Elastic Media,” Journal of the Acoustical Society of America, Vol. 74, No. 5, 1983, pp. 1642-1643. doi:10.1121/1.390131

[12] B. Singh and J. Arora, "Reflection of Plane Waves from a Free Surface of an Initially Stressed Transversely Isotropic Dissipative Medium,” Applied Mathematics, Vol. 2, 2011, pp. 115-125.

[13] B. Singh and J. Arora, "Reflection of Plane Waves from Free Surface of an Initially Stressed Rotating Orthotropic 
Dissipative Solid Half Space,” Engineering, Vol. 4, No. 3, 2012, pp. 170-175. doi:10.4236/eng.2012.43022

[14] M. M. Selim, "Reflection of Plane Waves at Free Surface of an Initially Stressed Dissipative Medium," World Academy of Science, Engineering and Technology, Vol. 30, 2008, pp. 120-127.
[15] T. Kong, D. X. Li and X. Wang, "Thermo-Magneto-Dynamic Stresses and Perturbation of Magnetic Field Vector in a Non-Homogeneous Hollow Cylinder," Applied Mathematical Modelling, Vol. 33, No. 11, 2009, pp. 29392950. doi:10.1016/j.apm.2008.10.003 